\title{
Trigemino-autonomic headache related to Gasperini syndrome
}

\author{
Zsófia Vesza · György Várallyay · Kristóf Szőke • \\ György Bozsik · Nóra Manhalter · Dániel Bereczki • \\ Csaba Ertsey
}

Received: 22 May 2010/Accepted: 12 August 2010/Published online: 29 August 2010

(C) Springer-Verlag 2010

\begin{abstract}
We report the association of ipsilateral trigemino-autonomic headache to a case of right-sided nuclear facial and abducens palsy (Gasperini syndrome), ipsilateral hypacusis and right hemiataxia, caused by the occlusion of the right anterior inferior cerebellar artery. Short-lasting attacks of mild to moderate ipsilateral fronto-periorbital head pain, accompanied by lacrimation and mild conjunctival injection during more severe attacks, were present from the onset of symptoms, with a gradual worsening over the next few months and remitting during naproxen therapy. Magnetic resonance imaging showed an infarct in the right cerebellar peduncle, extending toward the pontine tegmentum, also involving the ipsilateral spinal trigeminal nucleus and tract and the trigeminal entry zone. Gasperini syndrome may be accompanied by ipsilateral trigeminoautonomic head pain.
\end{abstract}

Keywords Gasperini syndrome - Trigemino-autonomic headache $\cdot$ Anterior inferior cerebellar artery $\cdot$ Infarction

Z. Vesza · K. Szőke · G. Bozsik · D. Bereczki · C. Ertsey ( $₫)$

Department of Neurology, Semmelweis University,

Balassa u. 6., 1083 Budapest, Hungary

e-mail: csaba.ertsey@gmail.com

G. Várallyay

MR Research Center, Szentágothai Knowledge Center,

Semmelweis University, Balassa u. 6., 1083 Budapest, Hungary

N. Manhalter

PhD Programme, Semmelweis University,

Budapest, Hungary

N. Manhalter

Department of Neurology, Nyírő Gyula Hospital,

Lehel u. 59., 1135 Budapest, Hungary

\begin{abstract}
Abbreviations
MRI Magnetic resonance imaging

AICA Anterior inferior cerebellar artery

CT Computed tomography

SUNCT Short-lasting unilateral neuralgiform headache attacks with conjunctival injection and tearing

MPR Multiplanar reconstructed (magnetic resonance imaging)
\end{abstract}

\section{Introduction}

Trigemino-autonomic cephalalgias (TACs) are a group of rare primary headaches in which the activation of the trigeminal and cranial parasympathetic pathways, and the consequent sympathetic dysfunction result in characteristic, strictly unilateral syndromes of head pain and autonomic accompaniments [1]. Not infrequently, a similar clinical picture can be caused by organic neurological diseases such as vascular disorders [2], tumors [2], multiple sclerosis [3] or viral encephalitis [4]. We present a case where the occlusion of the anterior inferior cerebellar artery (AICA) resulted in a characteristic brainstem syndrome (Gasperini syndrome) and a headache mimicking TAC.

\section{Case report}

A 77-year-old right-handed Caucasian man with a history of medically treated hypertension and hypercholesterolemia and no previous history of headache felt a sudden rotatory vertigo with associated vomiting and diarrhea 
during a trip to Italy. He was unable to stand and walk because of loss of balance, and the right side of his face felt paralyzed. The patient was admitted to the local hospital. Computed tomography confirmed an acute infarction of the right cerebellar hemisphere and multiple earlier supratentorial lacunar infarctions. Acetylsalicylic acid (100 mg daily) and a therapeutic dose of low molecular weight heparin were administered and the patient was transferred to our department for further evaluation.

On admission he complained about the clumsiness of the right hand, a moderate vertigo and a continuous mild pain in the right frontotemporal region irradiating toward the right ear. Attacks of moderate pain, accompanied by mild ipsilateral tearing and conjunctival injection, and lasting 0.5-3 min, could be provoked by touching the area of pain with no refractory periods between attacks. The neurological examination revealed right-sided nuclear type facial palsy, mild right abducens palsy and right-sided hypacusis. He had bilateral horizontal gaze-evoked nystagmus, which was more severe when looking to the right. He did not report double vision, and vertical eye movements were preserved. Hypalgesia, tactile and thermal hypesthesia were found on the right side of the face. A mild dysphagia and cerebellar dysarthria were noted; the soft palate and gag reflexes were preserved. There were no symptoms to suggest the involvement of the corticospinal and corticobulbar pathways. The sensory functions were intact on all limbs and the trunk. Dysdiadochokinesis, severe ataxia and dysmetria were found on the right-sided limbs. While sitting, the patient was falling to the right and was unable to stand or walk due to the vertigo.

Ultrasonography revealed mild artherosclerosis with no alteration of blood flow in the carotid and vertebral arteries. Magnetic resonance imaging (MRI) showed a subacute infarction in the inferolateral part of the right cerebellar hemisphere and in the right brachium pontis, extending toward the lateral portion of the pontine tegmentum, involving the spinal trigeminal nucleus and tract, and the trigeminal entry zone (Fig. 1a-c). MR-angiography showed atherosclerotic changes in the vertebral, basilar and carotid arteries, and the occlusion of the right AICA (Fig. 1d). The clinical picture was consistent with Gasperini syndrome. The headaches were diagnosed as 'Headache attributed to ischemic stroke (cerebral infarction)', ICHD-II code 6.1.1.; the close temporal relation between the stroke and the onset of headache precluded the diagnosis of a primary TAC [short-lasting unilateral neuralgiform headache attacks with conjunctival injection and tearing (SUNCT) syndrome]. During the 3-week-long inpatient period, the facial weakness showed a moderate improvement, but a mild right abducens and facial palsy, mild sensory disturbance of the face and significant ataxia in the right limbs persisted.
During the next months the patient experienced episodes of mild to moderate right frontal and periorbital pain, lasting $10 \mathrm{~s}$ to $5 \mathrm{~min}$, accompanied by ipsilateral tearing and conjunctival injection in about $30 \%$ of the attacks and recurring one to five times per day. Four months after the onset of symptoms headache severity and frequency started to increase gradually. Attacks of intense right fronto-periorbital head pain lasting 2-5 min and recurring 10 to 25 times a day were superimposed on a mild, constant right periorbital and parietal headache. Naproxen, taken on an as-needed basis, was helpful in reducing attack frequency. After 1 month the headaches became intolerable, causing insomnia. A detailed ophthalmological examination was negative. The neurological examination showed the residual symptoms of the previous infarction. Cranial MRI showed the previously noted infarct in the right AICA territory. Naproxen (275 mg twice a day) abolished the headaches, which recurred after the patient discontinued the medication. At the last follow-up visit, 18 months after the onset of symptoms, the patient was headache-free but still experienced a mild continuous right fronto-periorbital headache with moderately severe exacerbations lasting 2-5 min if naproxen was discontinued. His residual neurological symptoms were unchanged.

\section{Discussion}

Gasperini syndrome is a rare phenomenon, resulting from small ischemic or hemorrhagic lesions of the mediolateral tegmental pons; the core symptoms are caused by the involvement of the VIth and VIIth nerve nuclei or the intrapontine segments of these nerves [5]. The most common cause is the occlusion of the AICA. A recent review of the literature found only a few reports of this syndrome with a total of 13 patients including Gasperini's original case [6]. A further case was caused by pontine demyelination due to multiple sclerosis [7]. There may have been other cases in whom similar symptoms were not ascribed to Gasperini's name [8].

Beside the symptoms mentioned above, Gasperini's original report also described a contralateral hemisensory loss, caused by damage to the spinothalamic tract. This was seen in 11 of the 13 cases, making it the most common accompanying symptom. In some cases the trigeminothalamic tract was also involved, causing sensory disturbance on the contralateral half of the face. None of these were present in our patient. Ipsilateral facial sensory disturbance, suggesting the involvement of the spinal trigeminal tract or nucleus and hearing loss were also quite common $(8 / 13$ cases) - both were also part of our patient's symptoms. The ipsilateral headache involving the V/1 territory, one of the initial symptoms in our patient, had not been described by 
Fig. 1 Infarction in the right cerebellar peduncle extending toward the pontine tegmentum and trigeminal entry zone, caused by the occlusion of the right anterior inferior cerebellar artery (AICA). a axial

T2-weighted image, $\mathbf{b}$ axial and c sagittal MPR images showing the infarction which involves the trigeminal entry zone; on images $\mathbf{b}$ and $\mathbf{c}$ the trigeminal nerve is clearly visible (arrows). d MR-angiography showing the stenosis of the basilar artery (arrow) and occlusion of the right AICA, while the left AICA is clearly visible (arrowhead)
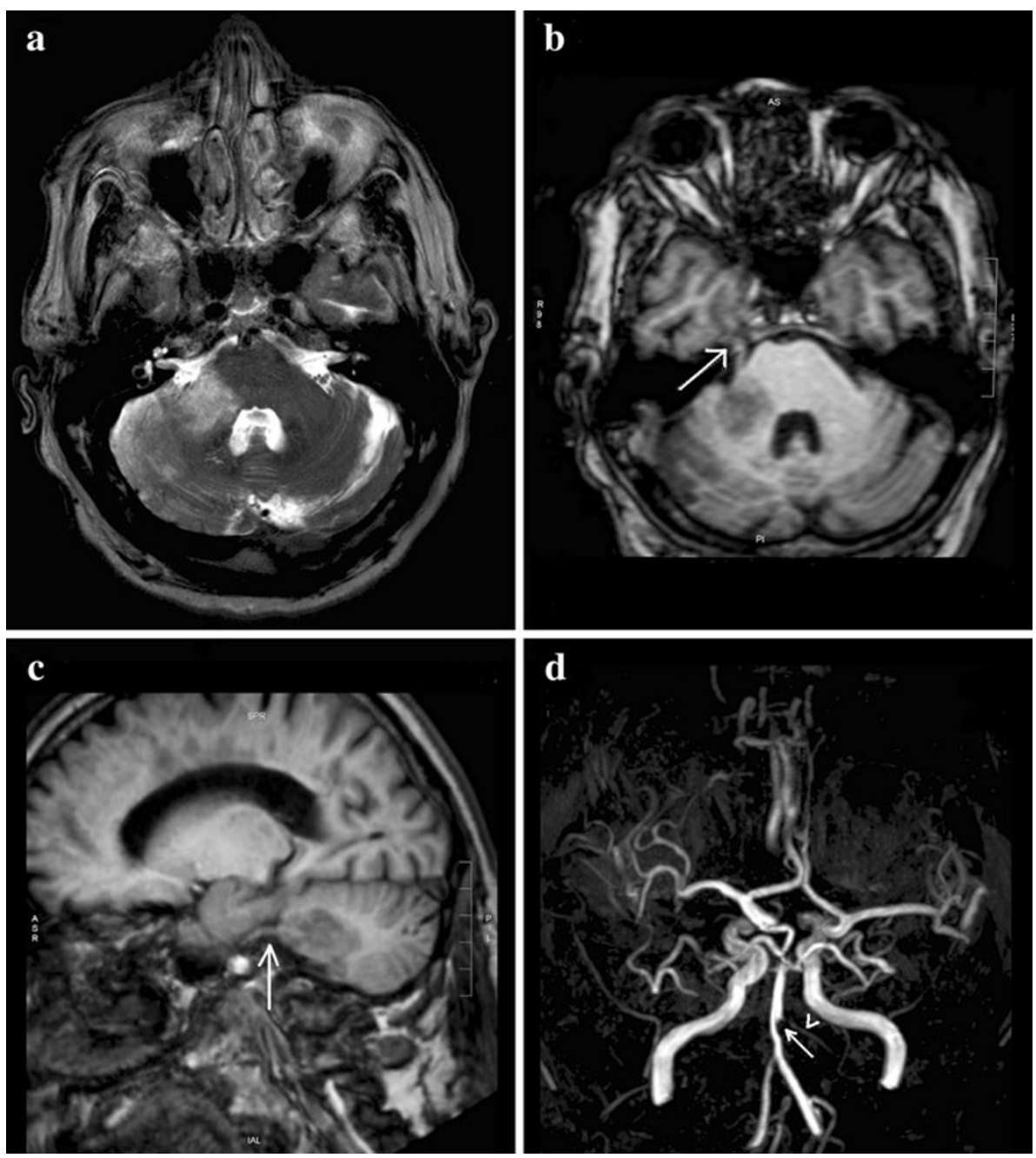

previous authors. The complaint of vertigo is also unusual. Taken together, our patient's symptoms indicate the involvement of nerves VI, VII and VIII (nuclear or intrapontine fibers), the trigeminal spinal tract or spinal tract nucleus, cochlear and vestibular nuclei or nerve, and middle cerebellar peduncle. The fact that the nerve VI palsy was not accompanied by double vision is probably due to the mild degree of the weakness and to the horizontal nystagmus which, through the consequent oscillopsia, may have influenced the patient's ability to discern the misaligned images on attempted right lateral gaze.

With the exception of a single case, all ischemic cases of Gasperini syndrome were caused by the occlusion of the AICA [6]. This artery supplies the lateral inferior pontine tegmentum and middle cerebellar peduncle, vestibulocochlear nerve including the root entry zone, inner ear, and anterior inferior cerebellum [9]. AICA infarcts are uncommon; in a prospective study they accounted for $0.9 \%$ of 770 acute strokes [9].

Infarcts in the posterior circulation territory are accompanied by headaches in more than $50 \%$ of cases [10]
Infarcts in the territory of the posterior inferior and superior cerebellar arteries are very frequently associated with headache which is one of the prominent symptoms [11].

On the other hand, AICA infarcts rarely cause headache: in a study describing 15 cases of AICA infarction not a single patient with headache as one of the symptoms was described [9]. In another study of 15 patients with multiple cerebellar and brainstem infarcts, none of the 5 patients with AICA infarcts reported headache, whereas 5 of the 10 other patients had headache as one of the presenting symptoms [12].

The patient's head pain changed a lot during the course of the disease. At the onset of symptoms the clinical picture was reminiscent of SUNCT syndrome, albeit with less prominent autonomic accompaniments. First division trigeminal neuralgia with tearing was a less probable option due to the lack of refractory periods between provoked attacks; a refractory period of $2-3 \mathrm{~min}$ is very frequent in trigeminal neuralgia, while the great majority of SUNCT patients in the case series of Pareja and Sjaastad [13] did not experience any refractory period after pain triggering. Later 
the patient's continuous mild head pain resembled hemicrania continua with superimposed severe attacks reminiscent of paroxysmal hemicrania. The sequential occurrence of different TAC types in the same patient has already been observed in some cases. One patient developed episodic paroxysmal hemicranias that later evolved in hemicrania continua [14]. Recently, Cosentino et al. [15] observed a patient in whom ipsilateral cluster headache, SUNCT and $\mathrm{HC}$ developed subsequently. These cases raise the issue of a common pathomechanism of these disorders, which most likely is the central disinhibition of the trigemino-autonomic reflex [16]. While in the primary TACs this disinhibition is probably mediated by hypothalamic activation (as already shown for cluster headache [17] and SUNCT syndrome [18]) through a hypothalamo-trigeminal pathway [19], the present case may have a different explanation.

The headaches caused by posterior circulation infarcts are very probably related to the activation of the trigeminovascular system [20]. In the present case, the involvement of the pontine trigeminal entry zone (which has been implicated in the pathogenesis of trigeminal neuralgia [21] and SUNCT-like headache [22]) may also have led to the attacks of pain and, during severe attacks, to autonomic symptoms by the activation of collaterals of second-order trigeminal neurones to the superior salivatory nucleus. Alternatively, the partial ischemic damage to the spinal trigeminal tract or nucleus (causing ipsilateral facial hypesthesia for pain and temperature) may have led to an aberrant signal transmission to higher-order pain centres.

\section{Conclusion}

Gasperini syndrome is a rare entity with a highly variable clinical picture. As the present case illustrates, ipsilateral headache involving the trigeminal territory may be one of its associated symptoms.

Consent The patient described has given written and verbal consent for his case to be published in this report.

Conflict of interest None.

\section{References}

1. Headache Classification Subcommittee of the International Headache Society (2004) The international classification of headache disorders. Cephalalgia 24(Suppl 1):1-160
2. Mainardi F, Trucco M, Maggioni F, Palestini C, Dainese F, Zanchin G (2010) Cluster-like headache. A comprehensive reappraisal. Cephalalgia 30:399-412. doi:10.1111/j.1468-2982. 2009.01993.x

3. Bogorad I, Blum S, Green M (2010) A case of MS presenting with SUNCT status. Headache 50(1):141-143. doi:10.1111/ j.1526-4610.2009.01514.x

4. Eguia P, Garcia-Monco JC, Ruiz-Lavilla N, Diaz-Konrad V, Monton F (2008) SUNCT and trigeminal neuralgia attributed to meningoencephalitis. J Headache Pain 9:51-53

5. Gasperini U (1912) Di un caso di emorragia protuberanziale. Contributo clinico allo studio delle sindromi pontine inferiori. Rif Med (Napoli) 28:880-898

6. Hayashi-Hayata M, Nakayasu H, Doi M, Fukada Y, Murakami T, Nakashima K (2007) Gasperini syndrome, a report of two cases. Intern Med 46:129-133

7. Krasnianski M, Müller T, Zierz S, Winterholler M (2009) Gasperini syndrome as clinical manifestation of pontine demyelination. Eur J Med Res 14:413-414

8. Roh JK, Kim BK, Chung JM (1999) Combined peripheral facial and abducens nerve palsy caused by caudal tegmental pontine infarction. Eur Neurol 41:99-102

9. Roquer J, Lorenzo JL, Pou A (1998) The anterior inferior cerebellar artery infarcts: a clinical-magnetic resonance imaging study. Acta Neurol Scand 97:225-230

10. Ferro JM, Melo TP, Oliveira V, Salgado AV, Crespo M, Canhao P (1995) A multivariate study of headache associated with ischemic stroke. Headache 35:315-319

11. Malm J, Kristensen B, Carlberg B, Fagerlund M, Olsson T (1999) Clinical features and prognosis in young adults with infratentorial infarcts. Cerebrovasc Dis 9:282-289

12. Terao S, Miura N, Osano Y, Takatsu S, Adachi K, Noda A, Sobue G (2005) Multiple cerebellar infarcts: clinical and pathophysiologic features. J Stroke Cerebrovasc Dis 14:193-198

13. Pareja JA, Sjaastad O (1997) SUNCT syndrome. A clinical review. Headache 37:195-202

14. Castellanos-Pinedo F, Zurdo M, Martínez-Acebes E (2006) Hemicrania continua evolving from episodic paroxysmal hemicrania. Cephalalgia 26:1143-1145

15. Cosentino G, Fierro B, Puma AR, Talamanca S, Brighina F (2010) Different forms of trigeminal autonomic cephalalgias in the same patient: description of a case. J Headache Pain $11: 281-284$

16. Goadsby PJ, Matharu MS, Boes CJ (2001) SUNCT syndrome or trigeminal neuralgia with lacrimation. Cephalalgia 21:82-83

17. May A, Bahra A, Buchel C, Frackowiak RSJ, Goadsby PJ (1998) Hypothalamic activation in cluster headache attacks. Lancet 351:275-278

18. May A, Bahra A, Buchel C, Turner R, Goadsby PJ (1999) Functional MRI in spontaneous attacks of SUNCT: short-lasting neuralgiform headache with conjunctival injection and tearing. Ann Neurol 46:791-793

19. Malick A, Burstein R (1998) Cells of origin of the trigeminohypothalamic tract in the rat. J Comp Neurol 400:125-144

20. Moskowitz MA (1984) The neurobiology of vascular head pain. Ann Neurol 16:154-168

21. Peker S, Akansel G, Sun I, Pamir NM (2004) Trigeminal neuralgia due to pontine infarction. Headache 44:1043-1045

22. De Benedittis G (1996) SUNCT syndrome associated with cavernous angioma of the brain stem. Cephalalgia 16:503-506 